\title{
Visualization and analysis of actin cytoskeleton organization in plants
}

\section{Gregory Pozhvanov}

Department of Plant Physiology and Biochemistry, Faculty of Biology, Saint Petersburg State University, Universitetskaya nab., 7-9, Saint Petersburg, 199034, Russian Federation

Address correspondence and requests for materials to Gregory Pozhvanov, g.pozhvanov@spbu.ru

\begin{abstract}
The plant cytoskeleton is a highly dynamic system that consists of two components: microfilaments and microtubules. Actin microfilaments are essential for polar growth, cytoplasmic streaming, directing polar growth, anchoring the nucleus, gravity sensing, signalling pathway integration and a number of other functions. Actin morphology and dynamics are orchestrated by a variety of small actin binding proteins, and some of them have become a source of actin interaction domains widely used as markers for microfilaments in fusions with fluorescent reporter proteins. However, older techniques are still employed for actin visualization. In this short review, we will focus on the diversity of fluorescent reporter fusions for F-actin and on approaches and existing free software for the analysis of cytoskeleton organization, mainly in Arabidopsis.
\end{abstract}

Keywords: cytoskeleton, actin, microfilament, plants, cell biology, visualization, microscopy, fluorescent protein, fluorescent dye.

\section{Introduction}

Every plant cell includes a number of different organelles, such as a nucleus, an endoplasmic reticulum, ribosomes, a Golgi apparatus, plastids, mitochondria, etc. However, these are not simply contained in the cellular envelope but are suspended in a network of fine filamentous structures - the cytoskeleton. The plant cytoskeleton consists of two main components, both built from protein polymers: microfilaments built from actin, and microtubules built from tubulin. The existence of intermediate filaments in plants is doubtful except for nuclear lamins, which exist but have sequences distinct from their animal analogues (Menzel, 1993). Microtubules (MTs) and microfilaments (MFs) are among the key elements of cytoarchitecture that define polarity in the plant cell (Medvedev, 2012) and direct the development of plant cell shape (Mathur, 2004; Klyachko, 2004). Microtubules and microfilaments feature intrinsic polarity since these are built from polymers of asymmetrical protein momomers. Although the very term 'cytoskeleton' suggests a rigid and stable thing, in reality the plant cytoskeleton is equal to the animal one in its dynamics (Steinborn, 2002; Paradez, Wright, and Ehrhardt, 2006). Both MTs and MFs are highly dynamic in vivo and are constantly rebuilt and rearranged. Furthermore, microtubule and microfilament subsets of the cytoskeleton mutually interact (Shevchenko, Kalinina, and Kordyum, 2007; Sampathkumar et al., 2011). However, functions of MTs and MFs differ in plant cells - MTs are involved in the formation of the mitotic spindle, cell shape establishment on par with MFs, and cell wall organization (Steinborn et al., 2002; Paradez, Wright, and Ehrhardt, 2006; Gutierrez et al., 2009; Sampathkumar et al., 2011). The actin microfilament network is essential for polar growth and intra- 
cellular communication: MFs mediate vesicle transport, cytoplasmic streaming, extension growth, anchoring of the nucleus, orientation of the cell division plane, gravity sensing, signalling of pathway integration, defense signalling, etc. (Baluška et al., 2001; Ketelaar et al., 2002; Klyachko, 2004; Bannigan and Baskin, 2005; Blancaflor, Wang, and Motes, 2006; Morita, 2010; Yoo et al., 2012; Blancaflor, 2013; Janda, Matoušková, Burketová, and Valentová, 2014). The actin cytoskeleton could be considered as a finer and more dynamic structure than MTs. In this review we will focus on MF organization, visualization approaches and the analysis of its organization.

\section{Actin cytoskeleton organization}

Microfilaments are $\approx 7-8 \mathrm{~nm}$ thick (Egelman, 1985; Holmes, Popp, Gebhard, and Kabsch, 1990) and are built from dimers of actin, which is found in the cytoplasm in two states, globular G-actin and polymeric F-actin, concentrations of which are in dynamic equilibrium. Microfilament is found in a steady state, at which the dissociation of G-actin from the "-" end and G-actin attachment to the "+" end are balanced (Staiger, Gibbon, Kovar, and Zonia, 1997; Blanchoin et al., 2010).

The actin cytoskeleton organization is vital for the establishment of cell shape, and its disruption leads to an irregular trichome shape, curvy root hairs, epidermal cell shape anomalies and an irregular shape of the hypocotyl. MFs carry the cytoplasmic strands and are responsible for cyclosis (Valster et al., 1997; Ketelaar et al., 2002). In the stele, root hairs and trichomes, actin microfilaments are assembled into thick bundles oriented along the cell's long axis. Thin actin microfilaments end next to the membranes and make contact with organelles (Ketelaar and Emons, 2001). Apical meristem cells lack thick bundles; their nuclei are located in the centre and are surrounded with a dense network of thin actin microfilaments. In the epiderm, a developed thin actin network underlies the plasma membrane (Mathur, J., Mathur, N., Kernebeck, and Hülskamp, 2003). Similarly, in root cap statocytes, the actin cytoskeleton lacks a developed fine MF network and is composed of short F-actin strands found near the plasma membrane or around organelles (Baluška and Hasenstein, 1997). The Arabidopsis genome consists of 10 genes of actin, and the majority of vegetative cells express ACT2 (Meagher and Fechheimer, 2003). However, various types of actin organization and its dynamics rely on a number of actin-binding proteins.

Actin microfilament branching at the angle of $70^{\circ}$ is facilitated by the ARP2/3 complex. It is essential for the development of trichome shape and epidermal cell lobes (Mathur, 2005). In these cells, the ARPC2 component of ARP2/3 mediates the interaction with microtubules (Havelková et al., 2015). Actin polymerizes into long MFs due to the formins (AtFH1) activity. Group I of these actin-binding proteins also enables actin cytoskeleton interaction with the plasma membrane and extracellular signals (Deeks et al., 2005). Fimbrins (AtFIM) carry two actin-binding domains (ABD1 and $\mathrm{ABD} 2)$, and each of them contains an EF-hand $\mathrm{Ca}^{2+}$-binding motif. Therefore, fimbrins are capable of MF cross-linking and inhibit their $\mathrm{Ca}^{2+}$-independent polymerization regulated by profilins (Higaki, Sano, and Hasezawa, 2007). Based on the second actin-binding domain of fimbrin (fABD2), various GFP fusions were developed to enable actin cytoskeleton in vivo visualization (Voigt et al., 2005). Vilins belong to the gelsolin family of actin-interacting proteins and facilitate actin bundling into thick bundles (Klahre et al., 2000). Profilins are small $(12-15 \mathrm{kDa})$ proteins that prevent the spontaneous polymerization of actin (Valster et al., 1997). Actin-depolymerizing factors (ADF) are also small $(15-20 \mathrm{kDa})$ proteins that are active in complex with actin-interacting protein 1 , severe MFs and further facilitate actin depolymerisation by making free ends in MFs (Ketelaar et al., 2004; Ketelaar, Anthony, and Hussey, 2004). Myosins are various motor proteins that enable organelle movement along the actin cytoskeleton and are essential for vesicular transport, assembly of transvacuolar actin cable, plasmodesmata pore adjustment, chloroplast positioning, and interaction between MTs and MFs (Šamaj, Peters, Volkmann, and Baluška, 2000; Meagher and Fecheimer, 2003; Grebe et al., 2003; Šamaj, Peters, Volkmann, and Baluška, 2006). Small GTP-binding proteins (GTPases) are also involved in the regulation of MF morphology in control of vesicular transport, polar growth and the development of complex cell morphology (Valster et al., 2000; Li et al., 2001; Vernoud et al., 2003; Berken et al., 2005; Li, Xu, J., Xu, Z., and Xue, 2005) as well as in reproduction development (Kawashima et al., 2014). ROPs convert external signals to the microfilament branching state by interaction with ARP2/3 (Klyachko, 2004; Xu and Scheres, 2005; Hussey, Ketelaar, and Deeks, 2006; Nagawa et al., 2012).

Rapid and dynamic rearrangement of the actin cytoskeleton is required for a number of cellular processes, e.g., gravitropism (Morita, 2010). For instance, SGR9 (SHOOT GRAVITROPISM 9) E3 ligase mediates the interaction of F-actin with amyloplasts (Nakamura et al., 2011). Vesicles carrying auxin efflux carriers of the PIN family are retargeted in a polar manner to a specific side of the cell membrane (Boutté et al., 2006). In this process of retrograde endocytosis, GNOM ( $\underline{A}$ DP-ribosylation factor - guanine nucleotide exchange factor) acts specifically at the assembly of vesicles containing PIN1 (Geldner et al., 2004), and GNL1 (GNOM-LIKE1) controls the trafficking of PIN2 (Kleine-Vehn et al., 2008). Both GNOM and GNL1 control auxin polar transport and at the same time are auxin-dependent (Dhonukshe et al., 2008; Nick, Han, and An, 2009). 
Clearly, various proteins interact with actin and modulate its morphology, dynamics and interaction with cell organelles and compartments. Therefore, molecular probes allowing the visualization of actin MFs, especially in vivo, and software tools facilitating the analysis of cytoskeleton organization, are required for a complete understanding of complex development patterns and responses to external stimuli.

\section{Actin cytoskeleton visualization}

Historically, the first methods used to visualize the actin cytoskeleton in plants under an optical microscope were staining of plant tissues with fluorescently labelled actin-binding phallotoxins after chemical fixation (Parthasarathy, Perdue, Witztum, and Alvernaz, 1985; Kakimoto and Shibaoka, 1987; Traas et al., 1987), and direct microinjection of such probes into cells (Cleary, 1995). In contrast to microtubules, which may be visualized by injection of fluorescently labelled tubulin, the applications of G-actin chemically derivatized in the same manner were not successful. However, a natural bicyclic hexapeptide phalloidin (Fig. 1A) from poisonous mushroom Amanita phalloides is capable of binding to actin - with a higher affinity to F-actin than to G-actin (Cooper, 1987). Therefore, upon addition to the cytoplasm, phalloidin selectively binds to actin MFs. A fluorescent dye such as rhodamine (Fig. 1B) or fluorescein (Fig. 1C), chemically bound to phalloidin, allows for fluorescent imaging of actin microfilaments in the cell in vivo or after chemical fixation (Fig. 2A). The choice between rhodamine and fluorescein (commonly fluorescein isothiocyanate, FITC) in a particular experiment is based on the fluorophores' excitation/emission properties: 540/565 $\mathrm{nm}$ for rhodamine phalloidin, and 496/516 nm for fluorescein phalloidin (Johnson, 2010); the set of available lasers for confocal imaging or filters for fluorescence imaging; as well as possible fluorescence cross-talk between fluorescent F-actin probe and autofluorescence of endogenous cellular structures such as the secondary cell wall. Importantly, phalloidin affects the G-/F-actin dynamic equilibrium (Dancker, Löw, Hasselbach, and Wieland, 1975). Its binding to F-actin stabilizes microfilaments and promotes G-actin polymerization, followed by the depletion of the G-actin pool. Therefore, when using phalloidin conjugates with fluorophores, it is important to keep its concentration in the staining solution minimal (or at least below the threshold level), otherwise the visualization itself may introduce artefacts to MF organization and/ or induce the formation of F-actin filaments of abnormal length. Since microinjection is not possible for tissues with small and numerous cells, a chemical fixation step (with formaldehyde and/or paraformaldehyde) is commonly added prior to staining to prevent phalloi-

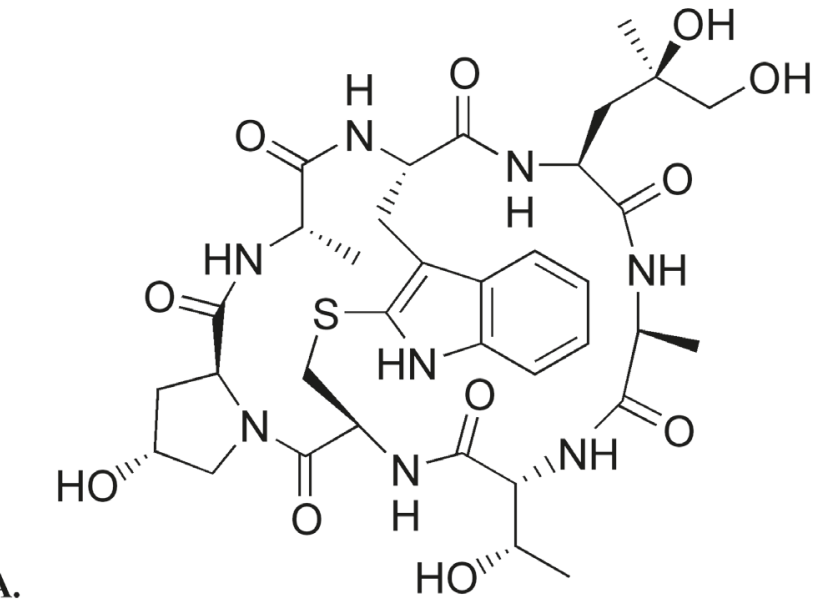

B.<smiles>CCN(CC)c1ccc2c(-c3ccccc3C(=O)O)c3ccc(=[N+](CC)CC)cc-3oc2c1</smiles>

C.<smiles>O=C(O)c1ccccc1-c1c2ccc(=O)cc-2oc2cc(O)ccc12</smiles>

Fig. 1. Chemical structures of phalloidin and fluorescent labels commonly used for F-actin visualization. A. Phalloidin from Amanita phalloides. B. Rhodamine. C. Fluorescein.

din-induced actin polymerization or actin cytoskeleton rearrangement resulting from the staining process. Typically, the sample preparation for MF phalloidin-based visualization includes chemical fixation, permeabilization of membranes with a detergent, and staining with rhodamine-phalloidin, for example (Van Gestel, Le, and Verbelen, 2001). Rhodamine-phalloidin is advantageous for labelling the actin cytoskeleton in plants where genetic transformation is hard or impossible, despite the rather long staining procedure it requires. For plant species in which genetic transformation is hard or unreliable, this method of actin MF visualization is still the only choice: actin has been visualized in lily pollen tubes (Hörmanseder, Obermeyer, and Foissner, 2005), tomato (Humbert et al., 2015), wheat (Khokhlova and Makarova, 2006) and other species. 
A.

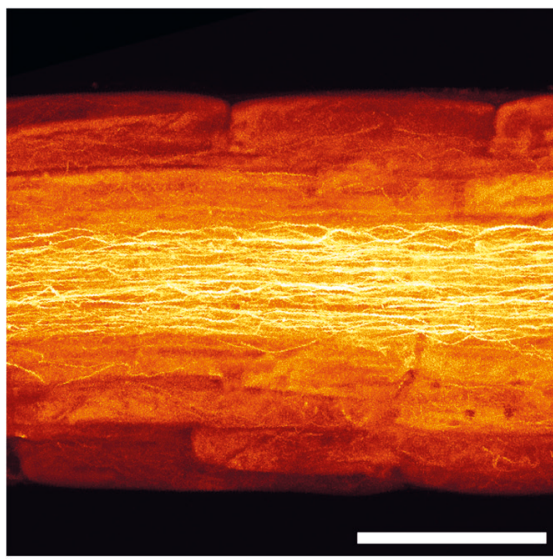

B.

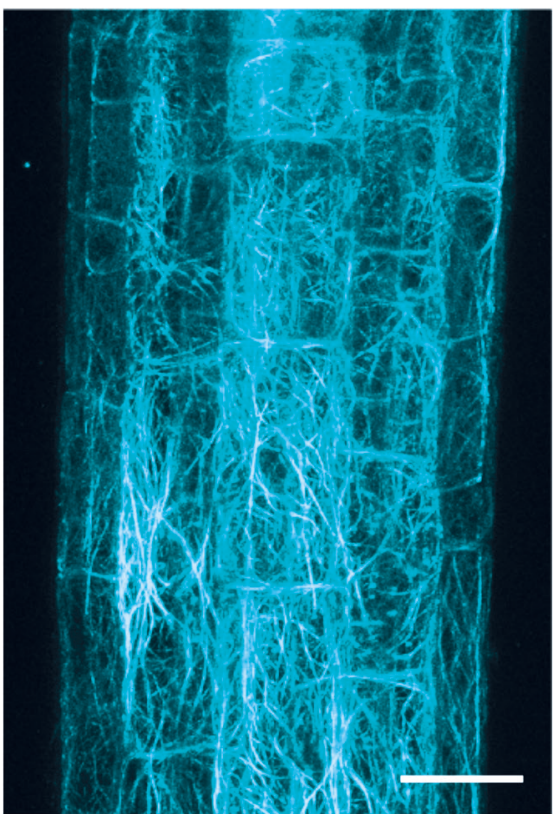

C.

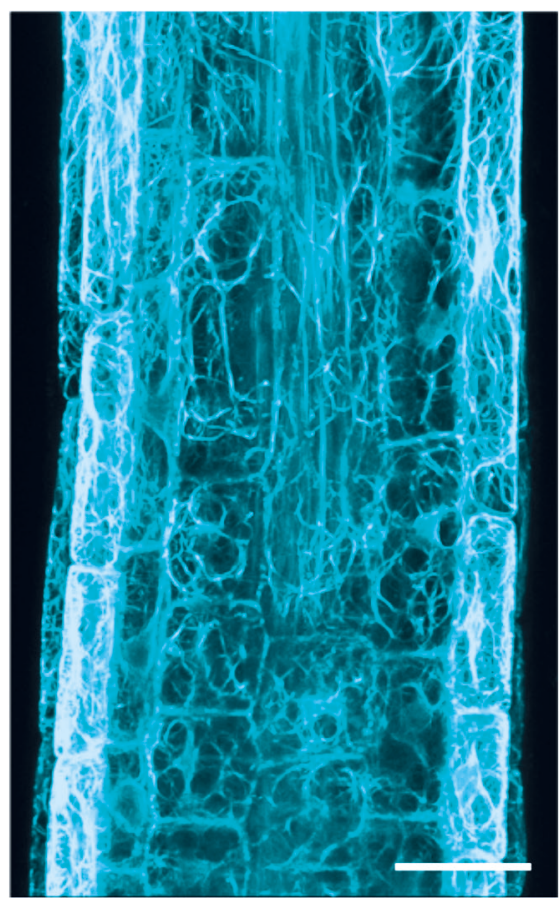

Fig. 2. Actin visualization in Arabidopsis root elongation zone. A. Staining with rhodamine-phalloidin. B. GFP-fABD2 reporter. C. Lifeact-Venus reporter. Scale bar: A, $50 \mu \mathrm{m} ; \mathrm{B}, \mathrm{C}: 20 \mu \mathrm{m}$.
Immunofluorescence labelling of the actin cytoskeleton became an appropriate technique for studying MFs in plant tissues or organs that are too thick or too large in diameter to be observed under a fluorescent or confocal microscope due to emission and fluorescence light absorption (Śniegowska-Świerk, Dubas, and Rapacz, 2015; Dyachok et al., 2016). In immunofluorescence labelling methodology, primary anti-actin antibodies are responsible for the specificity of binding, and secondary antibodies conjugated with fluorescent dye provide effective visualisation in situ. The procedure requires several additional steps, including obtaining tissue sections, cell wall digestion, permeabilization, incubation with primary antibodies, incubation with secondary antibodies, washing and mounting. Consecutively, the procedure of immunolabelling is complicated and may introduce artefacts as well, but for a number of plant objects it serves as the only option available. For an overview and a detailed protocol of visualization with immunofluorescence, please refer to the excellent paper by Dyachok and colleagues (Dyachok et al., 2016).

Any chemical fixative requires several minutes to penetrate the cells and react with protein polymers and halt the dynamic cytoskeleton. To overcome this problem, a cryofixation was developed (Lovy-Wheeler, Wilsen, Baskin, and Hepler, 2005). In this method, a plant specimen is first rapidly frozen in liquid gas such as propane $\left(\right.$ at $-180^{\circ} \mathrm{C}$ ), then freeze-substituted in dry acetone containing anhydrous glutaraldehyde, followed by further rehydratation and immunolabelling. In lily pollen tubes, this approach revealed a fringe of thin actin microfilaments near the pollen tube apex followed by numerous MFs in the pollen tube shank. The apical fringe of actin was otherwise destroyed by conventional chemical fixation methods (Lovy-Wheeler, Wilsen, Baskin, and Hepler, 2005). Then, using this image of the actin cytoskeleton organization as a reference, paper authors adjusted the chemical fixation method to room temperature to keep these pollen tube actin structures intact.

In plants where genetic transformation is well established or transient transformation is possible, a real breakthrough in in vivo cytoskeleton imaging started with the introduction of genetically encoded fusions of fluorescent proteins with natural actin-binding proteins from eukaryotic cells. A chimeric fusion protein composed of GFP (Green fluorescent protein) or its derivatives such as CFP (Cyan-), YFP (Yellow-), mOrange, mCherry, etc., fused with a whole actin-binding protein or its specific actin-binding domain, is constitutively expressed in transgenic plants, thus allowing direct visualization under a fluorescent or laser scanning confocal microscope without any general sample preparation steps (Kost, Spielhofer, and Chua, 1998; Schenkel et al., 2008; Dyachok et al., 2014). In GFP-mTn construct, 
the C-terminal actin-binding domain of mouse talin is responsible for the association of the probe with actin filaments (Kost, Spielhofer, and Chua, 1998). Unlike the full-length talin, its actin-binding protein does not trigger G-actin nucleation and was considered a robust marker tool. Indeed, colocalization of GFP-mTn-labelled actin with MFs labelled by rhodamine-phalloidin showed significant overlap in transiently transformed tobacco BY-2 cells (Kost, Spielhofer, and Chua, 1998). The introduction of GFP-mTn was encouraging. Its signal was stable and bright, thus facilitating live observation of actin organization and dynamics in transformed cells. Tobacco pollen tubes transiently transformed with GFP- $m T n$ revealed fine microfilaments in tube shank, and these pollen tubes featured the same growth rate and morphology as the control. However, it was demonstrated later that in transgenic GFP-mTn Arabidopsis lines, GFP-mTalin caused aberration in root hair growth (growth termination, swelling or thickening) and competed with ADF for the binding sites on MFs, thus inhibiting physiological depolymerisation of F-actin by ADF (Ketelaar, Anthony, and Hussey, 2004). The GFP-FABD2 construct was created to provide an alternative to GFP-mTn. In GFP-FABD2, GFP's C-terminus was fused to the second (C-terminal) F-actin binding domain of fimbrin, an actin-binding protein native to Arabidopsis (Sheahan, Staiger, Rose, and McCurdy, 2004; Voigt et al., 2005). This construct was active in different cell types of Arabidopsis ranging from root cap columella cells, rhizoderm to epidermal cells (Fig. 2B), mesophyll cells and trichomes, thus allowing research to overcome the limitations of full-length fimbrin fusions to GFP (Voitg et al., 2005). Double GFP - FABD2 fusion protein - GFP-FABD2-GFP - features an even stronger signal for actin cytoskeleton visualization (Wang, Yoo, and Blancaflor, 2008). A distinct actin-binding peptide sequence from yeast - ABP140 (Actin-binding peptide 140 ) - is another potent marker for actin cytoskeleton visualization. The essential part of ABP140, a peptide containing the first 17 amino acid residues, called Lifeact, is sufficient to replicate the F-actin affinity of the full-length ABP140 (Riedl et al., 2008). Lifeact fusions to GFP and, later, to Venus - an improved version of YFP with enhanced brightness and stability - which makes it possible to reveal fast F-actin dynamics in Arabidopsis (Fig. 2C) and Marchantia polymorpha moss in vivo (Era et al., 2009).

Since all GFP fusions developed to visualize actin in vivo are based on F-actin binding or interacting proteins, one could suspect that they somehow affect the normal physiology of actin microfilaments. Simply, the surface of an actin microfilament is finite, as well as the number of binding sites on it; therefore, if at least some of them are occupied by an effectively binding and constitutively expressed fluorescent protein fusion, the poten- tial for interactions of actin with its native cytoplasmic binding proteins becomes limited. In addition to that, GFP and its derivatives are capable of generating ROS under continuous light. For instance, drawbacks of GFP$\mathrm{mTn}$ application were already mentioned above. Similarly, other actin-binding fluorescent protein fusions were shown to imply some developmental or physiological alterations in their carrying plant. The GFP-FABD2-GFP fusion construct is bright and capable, but its use caused altered embryonal development, shorter and abnormally positioned root hairs and shorter branches in trichomes (Wang, Yoo, and Blancaflor, 2008). Lifeact-Venus was widely used for live F-actin observations, and it was generally believed to be safe for the dynamics of actin polymerization and depolymerisation (van der Honing, van Bezouwen, Emons, and Ketelaar, 2011). Surprisingly, Lifeact-Venus was recently demonstrated to interfere with nuclear actin (Du, Fan, Chen, and Feng, 2015). Also, Lifeact-Venus was found to delay actin bundles relocation and to reduce the cytoplasmic strand reorganization rate in epidermal cells of Arabidopsis (van der Honing, 2011), and, supporting this, in the same study Lifeact was shown to exchange more rapidly from actin MFs than FABD2. Dyachok et al. studied various protein fusion-based F-actin reporters for their potential impact on Arabidopsis development and morphology (2014). Their study showed that in general, FABD2-based fusions were less likely to cause abnormal F-actin bundling, which mTn-fusions were prone to do. Interestingly, the substitution of a strong constitutive $35 \mathrm{~S}$ promoter with UBQ10 promoter for GFP-FABD2-GFP probe prevented loss of fluorescence in the subsequent generations of transgenic plants and helped to avoid growth inhibitory effects of $35 \mathrm{~S}$ constructs. The expression level of marker fusion proteins appears to be very important for the correct development of polarly growing cells that have a shape highly dependent on the actin cytoskeleton, such as pollen tubes or root hairs. High expression levels of GFP-talin or GFP-fimbrin in tobacco pollen tubes led to the formation of abnormal transverse cortical hoops of actin, moving rings of $\mathrm{F}$-actin or its large aggregates (Wilsen et al., 2006). Our own data show that, for instance, GFP-FABD2 Arabidopsis plants exhibit slightly slower root growth pace but faster gravitropic bending development. Their metabolite profiles also differ from the background ecotype and accumulate higher levels of amino acids while being deficient in fatty acids, monoacyl glycerols and monosaccharides (unpublished data). Therefore, before making conclusions based on fusion fluorescent reporter protein visualization, related physiological parameters should be carefully considered and compared with results obtained by alternative reporters.

In addition to the actin cytoskeleton visualization techniques discussed above, a new and promising approach has recently emerged. Silicone-containing 
rhodamine derivatives such as SiR-methyl and SiR-carboxyl are bright, biocompatible and highly permeable (Lukinavičius et al., 2013). These fluorescent dyes may be easily conjugated with a marker protein or protein of interest, and since their emission spectra lies in near-infrared, they are excellent for super-resolution microscopy techniques. Later, SiR-actin and SiR-tubulin were generated and demonstrated to be effective in animal fibroblasts (Lukinavičius et al., 2014). However, no attempt has yet been made to apply these labels to plant systems.

\section{Analysis of actin cytoskeleton organization}

Given that the actin cytoskeleton plays a vital role in a number of physiological and developmental processes at the cellular level, it is important to qualitatively and quantitatively analyse and describe its organization. This includes the abundance of MFs, their preferential localization within the cell, length and angular distribution, thickness or diameter of bundles, branching, the presence and the number of contacts with the plasma membrane, etc. In a very simple approach, microfilaments are detected manually 'by eye' based on a fluorescence or confocal image and counted to provide quantitative data. Software filters such as threshold level in ImageJ (Schneider, Rasband, and Eliceiri, 2012) may provide aid in automated detection of microfilaments. Similarly, the thickness or diameter of actin cables could be measured indirectly in pixels based on a confocal image, and then converted to physical units, e.g., micrometers. This simple approach is robust yet time-consuming and is still employed (Zhang, Colyvas, Patrick, and Offler, 2017). However, sharp confocal images of a reasonable logical size are required for this approach. Predominantly longitudinal microfilaments found in root stele are not straight, however, and for their curvature assessment a simple index of curvature was suggested (Pozhvanov, Suslov, and Medvedev, 2013). This metric assumes that a curved microfilament will intersect a virtual longitudinal line grid more often than a straight one. However, the number of total intersections with longitudinal lines has to be normalized by the overall microfilament abundance in the cell, which is given by the number of intersections with a grid of transversal, or radial lines (Fig. 3):

$$
i_{\text {curv. }}=\frac{\sum_{i} n_{\text {axial }}}{\sum_{j} n_{\text {radial }}}
$$

In the living cell, the actin cytoskeleton is dynamic; it undergoes rearrangement to adjust to current cellular activity. Using fluorescent protein fusions to actin-binding proteins, MFs are trackable in real time. Captured time-course fluorescence images can be analysed further to quantify the actin cytoskeleton dynamics. This

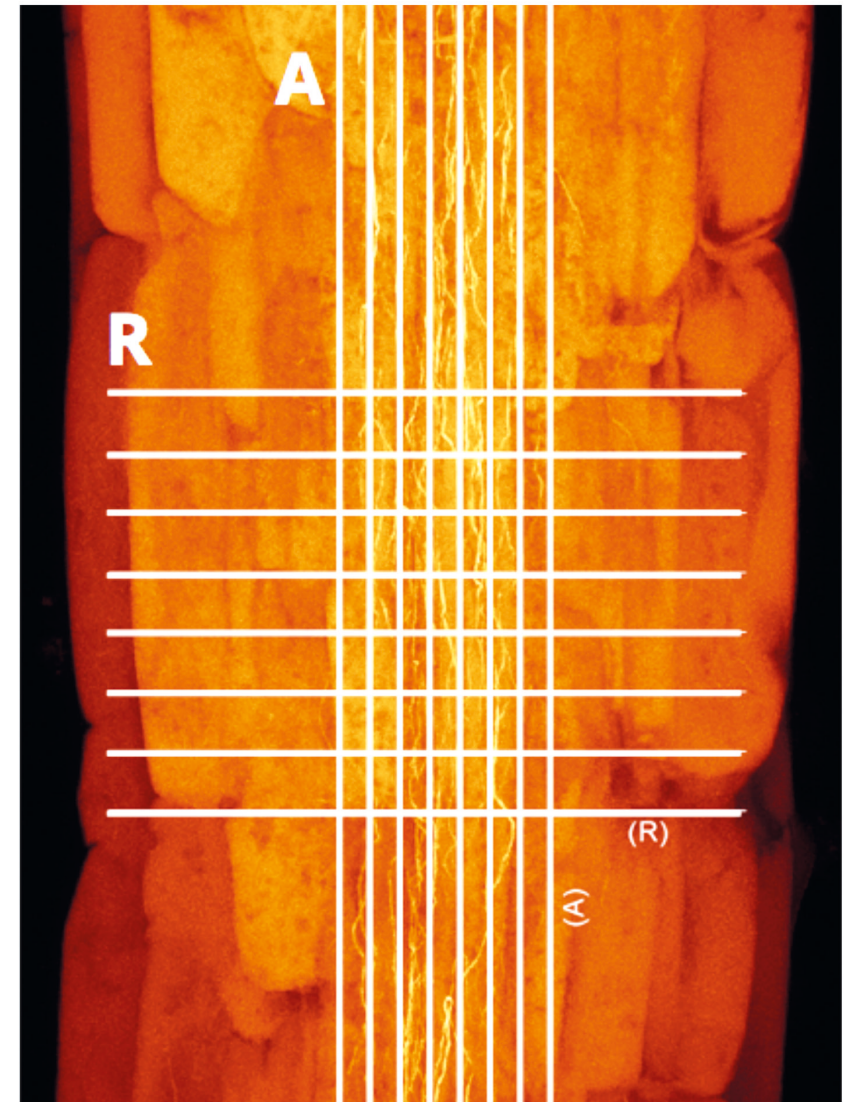

Fig. 3. Calculation of curvature index $\left(i_{\text {curv }}\right)$ for axially oriented microfilaments in root stele (wild-type Aranidopsis root stained with rhodamine-phalloidin). The number of actin microfilament intersections with axial grid lines is normalized by the number of intersections with radial grid lines (density of microfilaments).

type of analysis is commonly performed by automated pixel-to-pixel comparison of numerical fluorescence levels, $f_{x, y}$. If the given microfilament remains stable over time between two sequential key frames, its position in the digital image is the same, hence there is no significant shift in the level of signal: $f_{x, y}\left(t_{2}\right)-f_{x, y}\left(t_{1}\right)=0$. If a microfilament moves over time, the signal is lost at its initial position: $f_{x, y}\left(t_{2}\right)-f_{x, y}\left(t_{1}\right)<0$. Conversely, in case of a microfilament de novo assembly, a signal appears in previously dark pixels: $f_{x, y}\left(t_{2}\right)-f_{x, y}\left(t_{1}\right)>0$. The overall statistics of fluorescence shift gives an overview picture of actin dynamics within the cell (Nick, Han, and An, 2009).

To quantify the angular distribution, length and density of fibrillary structures such as MFs or MTs, a free dedicated software tool called Microfilament Analyzer (MFA) was developed (Jacques et al., 2013). Its analysis workflow is based on the concept of 'rotating polarisator. Virtual lines at a specific angle are projected on the image, and the luminosity (fluorescence signal) of adjacent pixels is compared along these lines. When the number of sequential pixels with brightness exceeding the threshold level reaches the minimal required length, 
A.

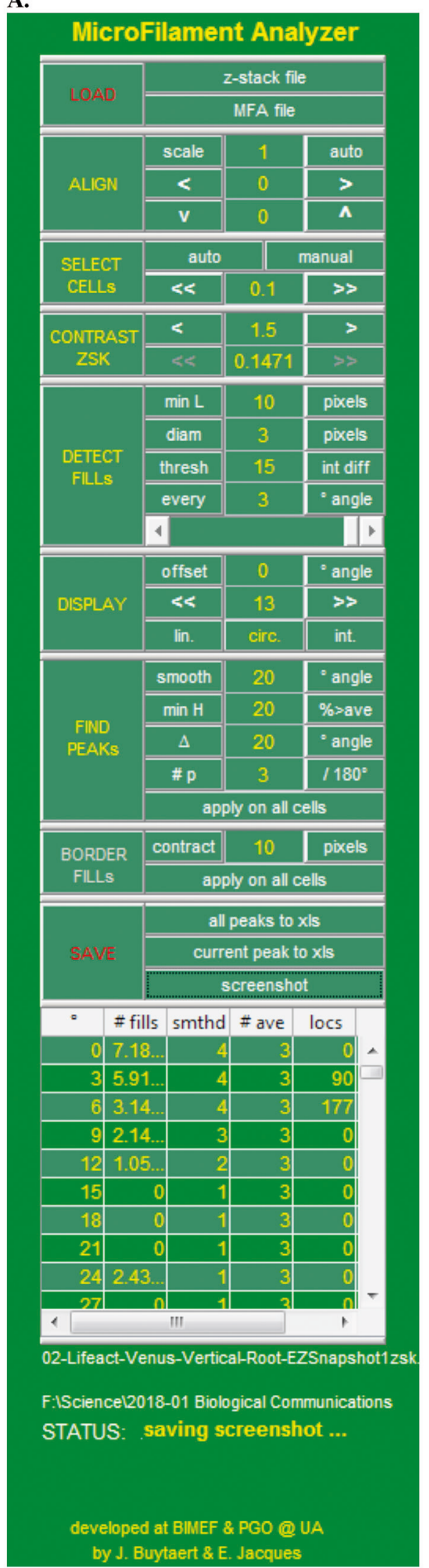

B.

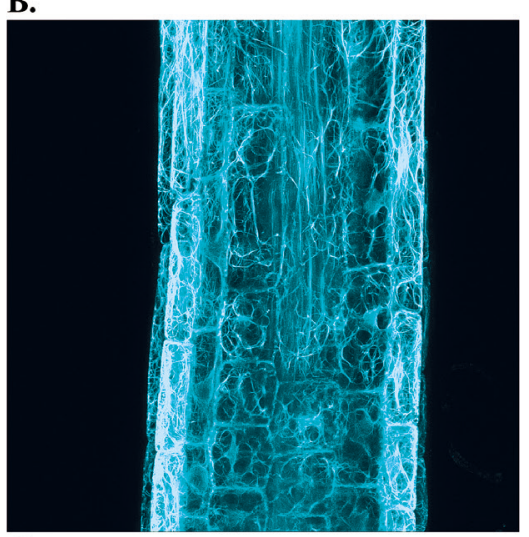

C.

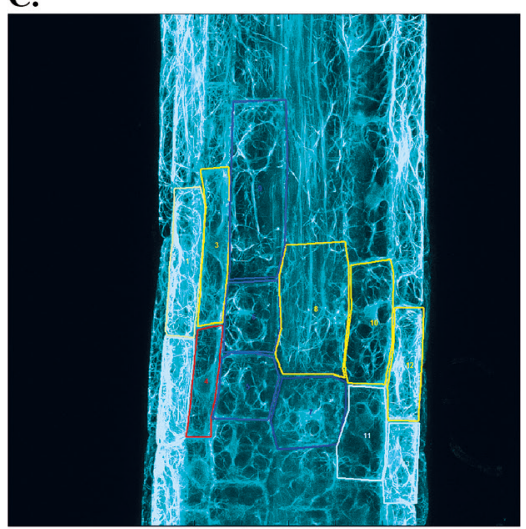

D.

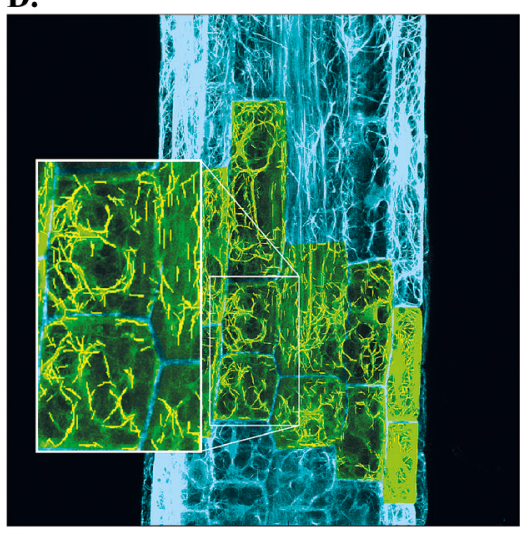

E.

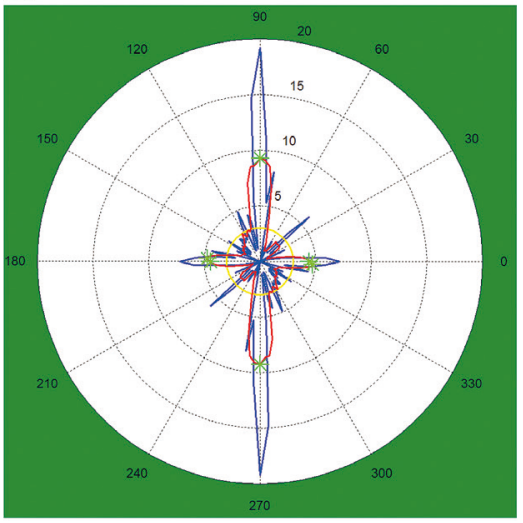

Fig. 4. Actin cytoskeleton analysis in Microfilament Analyzer software tool (Jacques et al., 2013). A. Analysis workflow: load z-stack file, adjust contrast for detection, apply settings for automatic filament detection, display analysis results. B. Image of Lifeact-Venus line of Arabidopsis thaliana loaded for analysis. C. Manual selecton of cell boundaries. D. Automatically detected microfilaments are highlighted in yellow. Inset: enlarged view of filaments detected in cells 5-6. E. Analysis results: microfilament angular distribution in a given cell. 
these count as a segment of microfilament of a specific length and orientation. After the detection of microfilaments oriented at a specific angle is completed, the virtual grid is rotated in a given angular step, and the detection process repeats (Fig. 4). MFA allows for the adjustment of image contrast, manual or automated cell boundary detection (based on image mask or other fluorescence channel, i.e., propidium iodide), and minimal length of MFs; their minimal thickness, threshold value and angular step are also adjustable parameters in the resolution of an image being analysed. The output of MFA is full statistics of MF angular distribution, number of filaments per cell, length of each detected filament, and the detection of predominant MF orientation angles. These statistics may be further classified or analysed to give numerical features of cytoskeleton rearrangement under stress or environmental response (Pozhvanov, Suslov, and Medvedev, 2016). The drawbacks of the current MFA version are its inability to batch process image and its operating system restriction (PC only).

An approach similar to MFA was introduced by Matouškova et al. (2014) to analyse actin cytoskeleton involvement in salicylate signalling. Confocal images were batch processed in ImageJ using a set of image masks of a given angular sector as low pass FFT filter for the detection of filamentous structures within the image (Matouškova et al., 2014, S1). The resulting images were converted to binary and subjected to particle analysis, resulting in statistics of length and number of filaments of a given angle.

Currently, a wide range of approaches and tools are available for various analyses of cytoskeleton organization and dynamics, and a number of new open source software will possibly emerge in the near future to describe and quantify the complex actin cytoskeleton features such as branching and interactions with actinbinding proteins or membranes.

\section{Conclusions}

The plant cytoskeleton is a dynamic system that is involved in various processes, ranging from cell housekeeping and division to complex responses to environmental stimuli. Over the past two decades, a number of visualization tools have been developed that literally cover the visible spectrum for fluorescence colour and allow us to reveal in vivo the structure and dynamics of the cytoskeleton using fluorescence and confocal microscopy. Since fluorescent protein fusion reporters utilize actin binding domains in their marker part, corresponding transgenic lines should be carefully checked for developmental and/or morphological anomalies. For plant objects unsuitable for transformation, other visualization methodology still exists, including staining with phalloidin-coupled dyes or immunofluorescence labelling. A number of software tools and approaches to quantify the cytoskeleton organization have been developed that allow for characterization of cytoskeleton rearrangements.

\section{References}

Baluška, F., and Hasenstein, K. H. 1997. Root cytoskeleton: its role in perception of and response to gravity. Planta 203:S69-S78. https://doi.org/10.1007/PL00008117

Baluška, F., Jasik, J., Edelmann, H. G., Salajova, T., and Volkmann, D. 2001. Latrunculin B-induced plant dwarfism: plant cell elongation is F-actin-dependent. Developmental Biology 231(1):113-124. https://doi.org/10.1006/ dbio.2000.0115

Bannigan, A. and Baskin, T.I., 2005. Directional cell expansion - turning toward actin. Current Opinion in Plant Biology 8(6):619-624. https://doi.org/10.1016/j. pbi.2005.09.002

Berken, A., Thomas, C., and Wittinghofer, A. 2005. A new family of RhoGEFs activates the Rop molecular switch in plants. Nature 436(7054):1176-1180. https://doi. org/10.1038/nature03883

Blancaflor, E. B. 2013. Regulation of plant gravity sensing and signaling by the actin cytoskeleton. American Journal of Botany 100(1):143-152. https://doi.org/10.3732/ ajb. 1200283

Blancaflor, E. B., Wang, Y. S., and Motes, C. M. 2006. Organization and function of the actin cytoskeleton in developing root cells. International Review of Cytology 252:219-264. https://doi.org/10.1016/S0074-7696(06)52004-2

Blanchoin, L., Boujemaa-Paterski, R., Henty, J. L., Khurana, P., and Staiger, C.J. 2010. Actin dynamics in plant cells: a team effort from multiple proteins orchestrates this very fast-paced game. Current Opinion in Plant Biology 13(6):714-723. https://doi.org/10.1016/j. pbi.2010.09.013

Boutté, Y., Crosnier, M. T., Carraro, N., Traas, J., and SatiatJeunemaitre, B. 2006. The plasma membrane recycling pathway and cell polarity in plants: studies on PIN proteins. Journal of Cell Science 119(7):1255-1265. https:// doi.org/10.1242/jcs.02847

Cleary, A. L. 1995. F-actin redistributions at the division site in living Tradescantia stomatal complexes as revealed by microinjection of rhodamine-phalloidin. Protoplasma 185(3):152-165. https://doi.org/10.1007/BF01272855

Cooper, J. A. 1987. Effects of cytochalasin and phalloidin on actin. The Journal of Cell Biology 105(4):1473-1478.

Dancker, P., Löw, I., Hasselbach, W., and Wieland, T. 1975. Interaction of actin with phalloidin: Polymerization and stabilization of F-actin. Biochimica et Biophysica Acta (BBA)-Protein Structure 400(2):407-414. https://doi. org/10.1016/0005-2795(75)90196-8

Deeks, M. J., Cvrcková, F., Machesky, L. M., Mikitová, V., Ketelaar, T., Zársky, V., Davies, B., and Hussey, P. J. 2005. Arabidopsis group le formins localize to specific cell membrane domains, interact with actin-binding proteins and cause defects in cell expansion upon aberrant expression. New Phytologist 168(3):529-540. https://doi. org/10.1111/j.1469-8137.2005.01582.x

Dhonukshe, P., Grigoriev, I., Fischer, R., Tominaga, M., Robinson, D. G., Hašek, J., Paciorek, T., Petrášek, J., Seifertová, D., Tejos, R., and Meisel, L. A. 2008. Auxin transport inhibitors impair vesicle motility and actin cytoskeleton dynamics in diverse eukaryotes. Proceedings of the $\mathrm{Na}$ tional Academy of Sciences, USA 105(11):4489-4494. https://doi.org/10.1073/pnas.0711414105 
Du, J., Fan, Y. L., Chen, T. L., and Feng, X. Q. 2015. Lifeact and Utr230 induce distinct actin assemblies in cell nuclei. Cytoskeleton 72(11):570-575. https://doi.org/10.1002/ $\mathrm{cm} .21262$

Dyachok, J., Paez-Garcia, A., Yoo, C. M., Palanichelvam, K., and Blancaflor, E. B. 2016. Fluorescence imaging of the cytoskeleton in plant roots; pp.139-153 in: Cytoskeleton Methods and Protocols: Methods and Protocols. https:// doi.org/10.1007/978-1-4939-3124-8 7

Dyachok, J., Sparks, J.A., Liao, F., Wang, Y.S., and Blancaflor, E. B. 2014. Fluorescent protein-based reporters of the actin cytoskeleton in living plant cells: Fluorophore variant, actin binding domain, and promoter considerations. Cytoskeleton 71(5):311-327. https://doi. org/10.1002/cm.21174

Egelman E. H. 1985. The structure of F-actin. Journal of Muscle Research and Cell Motility 6:129-151. https://doi. org/10.1007/BF00713056

Era, A., Tominaga, M., Ebine, K., Awai, C., Saito, C., Ishizaki, K., Yamato, K. T., Kohchi, T., Nakano, A. and Ueda, T., 2009. Application of Lifeact reveals F-actin dynamics in Arabidopsis thaliana and the liverwort, Marchantia polymorpha. Plant and Cell Physiology 50(6):1041-1048. https:// doi.org/10.1093/pcp/pcp055

Geldner, N., Richter, S., Vieten, A., Marquardt, S., TorresRuiz, R. A., Mayer, U., and Jürgens, G. 2004. Partial loss-of-function alleles reveal a role for GNOM in auxin transport-related, post-embryonic development of Arabidopsis. Development 131(2):389-400. https://doi. org/10.1242/dev.00926

Van Gestel, K., Le, J., and Verbelen, J. P. 2001. A comparison of F-actin labeling methods for light microscopy in different plant specimens: multiple techniques supplement each other. Micron 32(6):571-578. https://doi.org/10.1016/ S0968-4328(00)00054-8

Grebe, M., Xu, J., Möbius, W., Ueda, T., Nakano, A., Geuze, H. J., Rook, M. B., and Scheres, B. 2003. Arabidopsis sterol endocytosis involves actin-mediated trafficking via ARA6positive early endosomes. Current Biology 13(16):13781387. https://doi.org/10.1016/S0960-9822(03)00538-4

Gutierrez, R., Lindeboom, J. J., Paredez, A. R., Emons, A. M. C., and Ehrhardt, D. W. 2009. Arabidopsis cortical microtubules position cellulose synthase delivery to the plasma membrane and interact with cellulose synthase trafficking compartments. Nature Cell Biology 11(7):797-806. https://doi.org/10.1038/ncb1886

Havelková, L., Nanda, G., Martinek, J., Bellinvia, E., Sikorová, L., Šlajcherová, K., Seifertová, D., Fischer, L., Fišerová, J., Petrášek, J., and Schwarzerová, K. 2015. Arp2/3 complex subunit ARPC2 binds to microtubules. Plant Science 241:96-108. https://doi.org/10.1016/j. plantsci.2015.10.001

Higaki, T., Sano, T., and Hasezawa, S. 2007. Actin microfilament dynamics and actin side-binding proteins in plants. Current Opinion in Plant Biology 10(6):549-556. https:// doi.org/10.1016/j.pbi.2007.08.012

Holmes, K. C., Popp, D., Gebhard, W., and Kabsch, W. 1990. Atomic model of the actin filament. Nature 347:44-49. https://doi.org/10.1038/347044a0

Van der Honing, H. S. 2011. Actin-mediated cytoplasmic organization of plant cells. Wageningen University. 126 pp.

Van der Honing, H. S., van Bezouwen, L. S., Emons, A. M. C., and Ketelaar, T. 2011. High expression of Lifeact in Arabidopsis thaliana reduces dynamic reorganization of actin filaments but does not affect plant development. Cytoskeleton 68(10):578-587. https://doi.org/10.1002/cm.20534

Hormanseder K., Obermeyer G. and Foissner I. 2005. Disturbance of endomembrane trafficking by brefeldin $A$ and calyculin A reorganizes the actin cytoskeleton of Lilium longiflorum pollen tubes. Protoplasma 227:25-36. https://doi.org/10.1007/s00709-005-0132-4

Humbert C., Aimé S., Alabouvette C., Steinberg C., and Olivain C. 2015. Remodelling of actin cytoskeleton in tomato cells in response to inoculation with a biocontrol strain of Fusarium oxysporum in comparison to a pathogenic strain. Plant Pathology 64(6):1366-1374. https:// doi.org/10.1111/ppa.12375

Hussey, P. J., Ketelaar, T., and Deeks, M. J. 2006. Control of the actin cytoskeleton in plant cell growth. Annual Reviews in Plant Biology 57:109-125. https://doi.org/10.1146/annurev.arplant.57.032905.105206

Jacques, E., Buytaert, J., Wells, D. M., Lewandowski, M., Bennett, M. J., Dirckx, J., Verbelen, J.P., and Vissenberg, K. 2013. MicroFilament Analyzer, an image analysis tool for quantifying fibrillar orientation, reveals changes in microtubule organization during gravitropism. The Plant Journal 74(6):1045-1058. https://doi.org/10.1111/ tpj.12174

Janda, M., Matoušková, J., Burketová, L., and Valentová, O. 2014. Interconnection between actin cytoskeleton and plant defense signaling. Plant Signaling and Behavior 9(11):e976486. https://doi.org/10.4161/15592324.2014. 976486

Johnson, I.D. 2010. The Molecular Probes handbook. A guide to fluorescent probes and labeling technologies, 11 th Ed. Life Technologies Corporation, 1276 pp. ISBN: 0982927916

Kakimoto, T. and Shibaoka, H. 1987. A new method for preservation of actin filaments in higher plant cells. Plant and Cell Physiology 28(8):1581-1585. https://doi.org/10.1093/ oxfordjournals.pcp.a077453

Kawashima, T., Maruyama, D., Shagirov, M., Li, J., Hamamura, Y., Yelagandula, R., Toyama, Y., and Berger, F. 2014. Dynamic F-actin movement is essential for fertilization in Arabidopsis thaliana. Elife 3:e04501. https://doi. org/10.7554/eLife.04501

Ketelaar, T. and Emons, A. M. C. 2001. The cytoskeleton in plant cell growth: lessons from root hairs. New Phytologist 152(3):409-418. https://doi.org/10.1046/j.0028646X.2001.00278.x

Ketelaar, T., Allwood, E. G., Anthony, R., Voigt, B., Menzel, D., and Hussey, P.J. 2004. The actin-interacting protein AIP1 is essential for actin organization and plant development. Current Biology 14(2):145-149. https://doi. org/10.1016/j.cub.2004.01.004

Ketelaar, T., Anthony, R. G., and Hussey, P. J. 2004. Green fluorescent protein-mTalin causes defects in actin organization and cell expansion in Arabidopsis and inhibits actin depolymerizing factor's actin depolymerizing activity in vitro. Plant Physiology 136(4):3990-3998. https://doi. org/10.1104/pp.104.050799

Ketelaar, T., Faivre-Moskalenko, C., Esseling, J.J., de Ruijter, N.C., Grierson, C.S., Dogterom, M., and Emons, A. M. C. 2002. Positioning of nuclei in arabidopsis root hairs an actin-regulated process of tip growth. The Plant Cell 14(11):2941-2955. https://doi.org/10.1105/ tpc.005892

Khokhlova, L. P., and Makarova, M. V. 2006. Reorganizatsya tsitoskeleta pri dejstvii na rasteniya nizkih temperatur. [Reorganization of cytoskeleton under low temperature action in plants.] Uchenye zapiski Kazanskogo gosudarstvennogo universiteta 148(3):65-88.

Klahre, U., Friederich, E., Kost, B., Louvard, D., and Chua, N. H. 2000. Villin-like actin-binding proteins are expressed ubiquitously in Arabidopsis. Plant Physiology 122(1):3548. https://doi.org/10.1104/pp.122.1.35 
Kleine-Vehn, J., Łangowski, Ł., Wiśniewska, J., Dhonukshe, P. Brewer, P. B., and Friml, J. 2008. Cellular and molecular requirements for polar PIN targeting and transcytosis in plants. Molecular Plant 1(6):1056-1066. https://doi. org/10.1093/mp/ssn062

Klyachko, N. L. 2004. Actin cytoskeleton and the shape of the plant cell (a review). Russian Journal of Plant Physiology 51(6):827-833. https://doi.org/10.1023/ B:RUPP.0000047833.43849.ab

Kost, B., Spielhofer, P., and Chua, N. H. 1998. A GFP-mouse talin fusion protein labels plant actin filaments in vivo and visualizes the actin cytoskeleton in growing pollen tubes. The Plant Journal 16(3):393-401. https://doi.org/10.1046/ j.1365-313x.1998.00304.x

Li, H., Shen, J. J., Zheng, Z. L., Lin, Y., and Yang, Z. 2001. The Rop GTPase switch controls multiple developmental processes in Arabidopsis. Plant Physiology 126(2):670-684. https://doi.org/10.1104/pp.126.2.670

Li, L., Xu, J., Xu, Z. H., and Xue, H. W. 2005. Brassinosteroids stimulate plant tropisms through modulation of polar auxin transport in Brassica and Arabidopsis. The Plant Cell 17(10):2738-2753. https://doi.org/10.1105/ tpc.105.034397

Lovy-Wheeler, A., Wilsen, K. L., Baskin, T. I., and Hepler, P. K. 2005. Enhanced fixation reveals the apical cortical fringe of actin filaments as a consistent feature of the pollen tube. Planta 221(1):95-104. https://doi.org/10.1007/ s00425-004-1423-2

Lukinavičius, G., Reymond, L., D’este, E., Masharina, A., Göttfert, F., Ta, H., Güther, A., Fournier, M., Rizzo, S., Waldmann, H., and Blaukopf, C. 2014. Fluorogenic probes for live-cell imaging of the cytoskeleton. Nature Methods 11(7):731-733. https://doi.org/10.1038/Nmeth.2972

Lukinavičius, G., Umezawa, K., Olivier, N., Honigmann, A., Yang, G., Plass, T., Mueller, V., Reymond, L., Corrêa Jr, I. R., Luo, Z. G., and Schultz, C. 2013. A near-infrared fluorophore for live-cell super-resolution microscopy of cellular proteins. Nature Chemistry 5(2):132-139. https:// doi.org/10.1038/nchem.1546

Mathur, J. 2004. Cell shape development in plants. Trends in Plant Science 9(12):583-590. https://doi.org/10.1016/j. tplants.2004.10.006

Mathur, J. 2005. The ARP2/3 complex: giving plant cells a leading edge. BioEssays 27(4): 377-387. https://doi. org/10.1002/bies.20206

Mathur, J., Mathur, N., Kernebeck, B. and Hülskamp, M. 2003. Mutations in actin-related proteins 2 and 3 affect cell shape development in Arabidopsis. The Plant Cell 15(7):1632-1645. https://doi.org/10.1105/tpc.011676

Matoušková, J., Janda, M., Fišer, R., Šašek, V., Kocourková, D., Burketová, L., Dušková, J., Martinec, J., and Valentová, O. 2014. Changes in actin dynamics are involved in salicylic acid signaling pathway. Plant Science 223:36-44. https:// doi.org/10.1016/j.plantsci.2014.03.002

Meagher, R. B., and Fechheimer, M. 2003. The Arabidopsis cytoskeletal genome; pp. e0096 in: The Arabidopsis Book. https://doi.org/10.1199/tab.0096

Medvedev, S. S. 2012. Mechanisms and physiological role of polarity in plants. Russian Journal of Plant Physiology 59(4):502514. https://doi.org/10.1134/S1021443712040085

Menzel, D. 1993. Chasing coiled coils: intermediate filaments in plants. Plant Biology 106(4):294-300. https://doi. org/10.1111/j.1438-8677.1993.tb00751.x

Morita, M. T. 2010. Directional gravity sensing in gravitropism. Annual Review of Plant Biology 61:705-720. https:// doi.org/10.1146/annurev.arplant.043008.092042

Nagawa, S., Xu, T., Lin, D., Dhonukshe, P., Zhang, X., Friml, J., Scheres, B., Fu, Y., and Yang, Z. 2012. ROP GTPase-depen- dent actin microfilaments promote PIN1 polarization by localized inhibition of clathrin-dependent endocytosis. PLoS Biology 10(4):e1001299. https://doi.org/10.1371/ journal.pbio.1001299

Nakamura, M., Toyota, M., Tasaka, M., and Morita, M. T. 2011. An Arabidopsis E3 ligase, SHOOT GRAVITROPISM9, modulates the interaction between statoliths and F-actin in gravity sensing. The Plant Cell 23(5):1830-1848. https:// doi.org/10.1105/tpc.110.079442

Nick, P., Han, M. J., and An, G. 2009. Auxin stimulates its own transport by shaping actin filaments. Plant Physiology 151(1):155-167. https://doi.org/10.1104/pp.109.140111

Paradez, A., Wright, A., and Ehrhardt, D. W. 2006. Microtubule cortical array organization and plant cell morphogenesis. Current Opinion in Plant Biology 9(6):571-578. https:// doi.org/10.1016/j.pbi.2006.09.005

Parthasarathy, M. V., Perdue, T.D., Witztum, A., and Alvernaz, J. 1985. Actin network as a normal component of the cytoskeleton in many vascular plant cells. American Journal of Botany 72(8):1318-1323. https://doi. org/10.1002/j.1537-2197.1985.tb08386.x

Pozhvanov, G. A., Gobova, A. E., Bankin, M. P., Vissenberg, K., and Medvedev, S. S. 2016. Ethylene is involved in the actin cytoskeleton rearrangement during the root gravitropic response of Arabidopsis thaliana. Russian Journal of Plant Physiology 63(5):587-596. https://doi.org/10.1134/ S1021443716050095

Pozhvanov, G.A., Suslov, D. V., and Medvedev, S. S. 2013. Actin cytoskeleton rearrangements during the gravitropic response of Arabidopsis roots. Cell and Tissue Biology 7(2):185-191. https://doi.org/10.1134/ S1990519X13020120

Riedl, J., Crevenna, A. H., Kessenbrock, K., Yu, J.H., Neukirchen, D., Bista, M., Bradke, F., Jenne, D., Holak, T. A., Werb, Z., and Sixt, M. 2008. Lifeact: a versatile marker to visualize F-actin. Nature Methods 5(7):605-607. https:// doi.org/10.1038/nmeth.1220

Šamaj, J., Chaffey, N., Tirlapur, U., Jasik, J., Hlavacka, A., Cui, Z., Volkmann, D., Menzel, D., and Baluska, F. 2006. Actin and myosin VIII in plant cell-cell channels; pp. 119134 in: Cell-Cell Channels. Springer: New York. https:// doi.org/10.1007/978-0-387-46957-7_8

Šamaj, J., Peters, M., Volkmann, D., and Baluška, F. 2000. Effects of myosin ATPase inhibitor 2, 3-butanedione 2-monoxime on distributions of myosins, F-actin, microtubules, and cortical endoplasmic reticulum in maize root apices. Plant and Cell Physiology 41(5):571-582. https://doi.org/10.1093/pcp/41.5.571

Sampathkumar, A., Lindeboom, J. J., Debolt, S., Gutierrez, R., Ehrhardt, D. W., Ketelaar, T., and Persson, S. 2011. Live cell imaging reveals structural associations between the actin and microtubule cytoskeleton in Arabidopsis. The Plant Cell 23(6):2302-2313. https://doi.org/10.1105/ tpc.111.087940

Schneider, C. A., Rasband, W. S., and Eliceiri, K. W. 2012. NIH Image to Image): 25 years of image analysis. Nature Methods 9(7):671-675. https://doi.org/10.1038/nmeth.2089

Sheahan, M. B., Staiger, C. J., Rose, R. J., and McCurdy, D. W. 2004. A green fluorescent protein fusion to actin-binding domain 2 of Arabidopsis fimbrin highlights new features of a dynamic actin cytoskeleton in live plant cells. Plant Physiology 136(4):3968-3978. https://doi.org/10.1104/ pp.104.049411

Schenkel, M., Sinclair, A. M., Johnstone, D., Bewley, J. D., and Mathur, J. 2008. Visualizing the actin cytoskeleton in living plant cells using a photo-convertible mEos::FABDmTn fluorescent fusion protein. Plant Methods 4:21. https://doi.org/10.1186/1746-4811-4-21 
Shevchenko, G. V., Kalinina, Y. M., and Kordyum, E. L. 2007. Interrelation between microtubules and microfilaments in the elongation zone of Arabidopsis root under clinorotation. Advances in Space Research 39(7):1171-1175. https://doi.org/10.1016/j.asr.2007.02.072

Śniegowska-Świerk, K., Dubas, E., and Rapacz, M. 2015. Drought-induced changes in the actin cytoskeleton of barley (Hordeum vulgare L.) leaves. Acta Physiologiae Plantarum 37:73. https://doi.org/10.1007/s11738-015-1820-0

Staiger, C.J., Gibbon, B.C., Kovar, D.R., and Zonia, L.E. 1997. Profilin and actin-depolymerizing factor: modulators of actin organization in plants. Trends in Plant Science 2(7):275-281. https://doi.org/10.1016/S13601385(97)86350-9

Steinborn, K., Maulbetsch, C., Priester, B., Trautmann, S., Pacher, T., Geiges, B., Küttner, F., Lepiniec, L., Stierhof, Y. D., Schwarz, H., and Jürgens, G. 2002. The Arabidopsis PILZ group genes encode tubulin-folding cofactor orthologs required for cell division but not cell growth. Genes and Development 16(8):959-971. https://doi.org/10.1101/ gad.221702

Traas, J. A., Doonan, J. H., Rawlins, D. J., Shaw, P. J., Watts, J., and Lloyd, C. W. 1987. An actin network is present in the cytoplasm throughout the cell cycle of carrot cells and associates with the dividing nucleus. The Journal of Cell Biology 105(1):387-395. https://doi.org/10.1083/jcb.105.1.387

Valster, A. H., Pierson, E.S., Valenta, R., Hepler, P. K., and Emons, A. M. C. 1997. Probing the plant actin cytoskeleton during cytokinesis and interphase by profilin microinjection. The Plant Cell 9(10):1815-1824. https://doi. org/10.1105/tpc.9.10.1815

Vernoud, V., Horton, A. C., Yang, Z., and Nielsen, E. 2003. Analysis of the small GTPase gene superfamily of Ara- bidopsis. Plant Physiology 131(3):1191-1208. https://doi. org/10.1104/pp.013052

Voigt, B., Timmers, A. C., Šamaj, J., Müller, J., Baluška, F., and Menzel, D. 2005. GFP-FABD2 fusion construct allows in vivo visualization of the dynamic actin cytoskeleton in all cells of Arabidopsis seedlings. European Journal of Cell Biology 84(6):595-608. https://doi.org/10.1016/j. ejcb.2004.11.011

Wang, Y. S., Yoo, C. M., and Blancaflor, E. B. 2008. Improved imaging of actin filaments in transgenic Arabidopsis plants expressing a green fluorescent protein fusion to the $\mathrm{C}$-and $\mathrm{N}$-termini of the fimbrin actin-binding domain 2. New Phytologist 177(2):525-536. https://doi. org/10.1111/j.1469-8137.2007.02261.x

Wilsen, K. L., Lovy-Wheeler, A., Voigt, B., Menzel, D., Kunkel, J. G., and Hepler, P. K. 2006. Imaging the actin cytoskeleton in growing pollen tubes. Sexual Plant Reproduction 19(2):51-62. https://doi.org/10.1007/s00497-0060021-9

$\mathrm{Xu}$, J. and Scheres, B. 2005. Cell polarity: ROPing the ends together. Current Opinion in Plant Biology 8(6):613-618. https://doi.org/10.1016/j.pbi.2005.09.003

Yoo, C. M., Quan, L., Cannon, A. E., Wen, J., and Blancaflor, E. B. 2012. AGD1, a class 1 ARF-GAP, acts in common signaling pathways with phosphoinositide metabolism and the actin cytoskeleton in controlling Arabidopsis root hair polarity. The Plant Journal 69(6):1064-1076. https://doi. org/10.1111/j.1365-313X. 2011.04856.x

Zhang, H. M., Colyvas, K., Patrick, J. W., and Offler, C. E. 2017. $\mathrm{A} \mathrm{Ca}^{2+}$-dependent remodelled actin network directs vesicle trafficking to build wall ingrowth papillae in transfer cells. Journal of Experimental Botany 68(17):4749-4764. https://doi.org/10.1093/jxb/erx315 